\title{
Stabilization of the Unicycle with Rider
}

\author{
Dmitry V. Zenkov ${ }^{1}$ \\ Department of Mathematics \\ University of Michigan \\ Ann Arbor, MI 48109 \\ zenkov@math.1sa.umich.edu
}

\author{
Anthony M. Bloch ${ }^{2}$ \\ Department of Mathematics \\ University of Michigan \\ Ann Arbor, MI 48109 \\ abloch@math.1sa.umich.edu
}

\author{
Jerrold E. Marsden ${ }^{3}$ \\ Control and Dynamical Systems \\ California Institute of Technology 107-81 \\ Pasadena, CA 91125 \\ marsden@cds.caltech.edu \\ Proc CDC, 38, (1999), 3470-3471.
}

\begin{abstract}
In this paper we discuss stabilization of a nonholonomic system consisting of a unicycle with rider. We show in particular that one can achieve stability of slow steady vertical motions by imposing a feedback control force on the rider's limb.
\end{abstract}

\section{Introduction}

In this paper we study the stabilization problem for a model of a rider on a unicycle. We use some of the ideas discussed in [10], where various techniques for studying the stability of motion of nonholonomic mechanical systems are analyzed. In particular, energybased methods are considered as well as use of the socalled Lyapunov-Malkin theorem.

We model the rider on a unicycle by a double pendulum on a wheel, the two pendula representing the body and the limb of the rider. This leads to complicated but tractable equations. See [11] for details. To achieve stabilization of slow vertical steady state motions of the unicycle we then apply linear control to the pendulum representing the limb of the rider. The stability of the overall system is tested using the Lyapunov-Malkin theorem. This theorem, which enables us to conclude overall nonlinear stability using partial spectral information about the system, turns out to be particularly useful for the analysis of nonholonomic systems (see

\footnotetext{
${ }^{1}$ Research partially supported by NSF grant DMS-9803181, AFOSR grant F49620-96-1-0100, a University of Michigan Rackham Fellowship, and an NSF group infrastructure grant at the University of Michigan

${ }^{2}$ Research partially supported by NSF grant DMS-9803181, AFOSR grant F49620-96-1-0100, and an NSF group infrastructure grant at the University of Michigan

${ }^{3}$ Research partially supported by AFOSR grant F49620-95-10419
}

$[5,8,10])$. Fast motions may also be stabilized and are in fact easier to handle because of the stabilizing effect of the wheel velocity.

While the analysis here is quite nontrivial in itself we intend to extend it both to more complex nonholonomic/robotic systems and to more complicated nonlinear control techniques; for example, the matching control technique discussed in $[2,3]$.

\section{Modeling the Unicycle with Rider}

We discuss here the dynamics of a homogeneous disk on a horizontal plane with a double pendulum attached. The upper pendulum is free to move in the plane orthogonal to the disk while the lower pendulum stays "vertical" in the disk's plane. We view this as a simple model of a rider on a unicycle. The configuration space with coordinates $(\theta, \varkappa, \psi, \phi, x, y)$ is $S^{1} \times S^{1} \times S^{1} \times S E(2)$. See Figure 1 for details. This mechanical system is

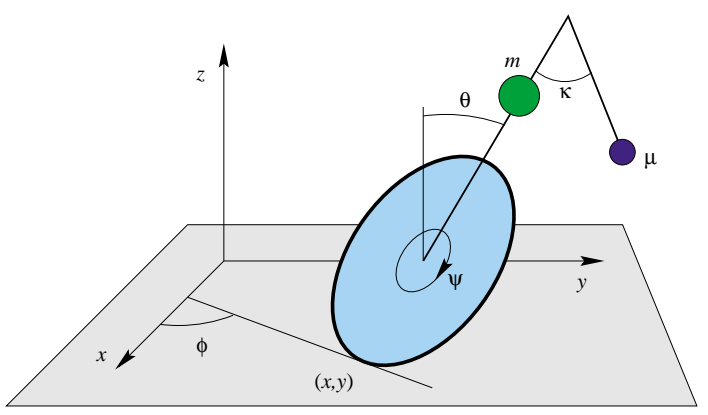

Figure 1: The disk on the horizontal plane.

$S O(2) \times S E(2)$-invariant. The action by the group element $(\alpha, \beta, a, b)$ on the configuration space is given by $(\theta, \varkappa, \psi, \phi, x, y) \mapsto(\theta, \varkappa, \psi+\alpha, \phi+\beta, x \cos \beta-y \sin \beta+$ $a, x \sin \beta+y \cos \beta+b)$. 
To stabilize slow vertical steady state motions of the unicycle with rider moving along a straight line, we introduce a single linear control

$$
u=k_{1} \theta+k_{2} \varkappa+k_{3} \dot{\theta}+k_{4} \dot{\varkappa}
$$

with gain parameters $\left(k_{1}, k_{2}, k_{3}, k_{4}\right)$ into the upper pendulum. One can think of this as a controlled limb of the rider. We remark that fast steady state motions of the unicycle without rider do not require stabilization (see [10]). It is this fact that makes fast motions of the unicycle with rider easier to stabilize than slow motions.

\section{Feedback Stabilization}

Using results of [10], we find the special local coordinates $(r, s)$ in the neighborhood of an upright steady state motion of the unicycle in which the dynamics of the reduced system is governed by the equations

$$
\dot{r}=A_{k} r+R(r, s), \quad \dot{s}=S(r, s),
$$

where $A_{k}$ is a $4 \times 4$ matrix, $s$ is a two dimensional vector, and $R(r, s), S(r, s)$ represent nonlinear terms vanishing when $r=0$. The entries of the matrix $A_{k}$ depend on the gain parameters. The steady state motions are the equilibria

$$
r=0, \quad s=c
$$

of (1). The equilibrium $r=0, s=0$ represents the steady state motion under consideration while equilibria (2) with $c \neq 0$ represent nearby steady state motions.

According to the Lyapunov-Malkin theorem $[6,7]$, the equilibria (2) are stable for small values of $c$ if all eigenvalues of the matrix $A_{k}$ have negative real parts. We emphasize the importance of the fact that $R(0, s)=0, S(0, s)=0$. This theorem has been used by a number of authors in analyzing stability of nonholonomic systems. See $[5,8,10]$ and references therein. In particular, we stress that the conditions $R(0, s)=0$ and $S(0, s)=0$ are valid for all systems considered in $[1,10]$.

In the next theorem we prove existence of the linear feedback control that forces the spectrum of the matrix $A_{k}$ to belong to the left half plane.

Theorem. There exists an open non-empty stability region $\mathcal{S}$ in the space of the gain parameters. For any $\left(k_{1}, k_{2}, k_{3}, k_{4}\right) \in \mathcal{S}$ the spectrum of $A_{k}$ belongs to the left half plane and therefore by the Lyapunov-Malkin theorem the steady state motion $r=0, s=0$ is stable.

The proof of this theorem is based on the RouthHurwitz analysis of the characteristic polynomial of the matrix $A_{k}$. For details see [11].

\section{Final Remarks}

We emphasize that the Lyapunov-Malkin theorem can be used for nonlinear feedback stabilization. It extends a spectral stability condition to a nonlinear setting. We expect that the domain of the local coordinates $(r, s)$ can be expanded by an appropriate choice of nonlinear control terms. The basin of attraction therefore may be enlarged. We intend to address this issue in a future publication.

\section{References}

[1] Bloch, A.M., P.S. Krishnaprasad, J.E. Marsden, \& R. Murray, Nonholonomic Mechanical Systems with Symmetry, Arch. Rat. Mech. An. 136, 1996, 21-99.

[2] Bloch, A.M., N. Leonard, \& J.E. Marsden, Stabilization of Mechanical Systems Using Controlled Lagrangians, Proc. CDC 36, 1997, 2356-2361.

[3] Bloch, A.M., N. Leonard, \& J.E. Marsden, Matching and Stabilization by the Method of Controlled Lagrangians, Proc. CDC 37, 1998, 1446-1451.

[4] Bloch, A.M., M. Reyhanoglu, \& N.H. McClamroch, Control and Stabilization of Nonholonomic Dynamic Systems, IEEE Trans. Autom. Contr. 37, 1992, $1746-1757$.

[5] Karapetyan, A.V., On Stability of Steady State Motions of a Heavy Solid Body on an Absolutely Rough Horizontal Plane, J. Appl. Math. Mech. 45, 1981, 604608.

[6] Lyapunov, A.M., The General Problem of the Stability of Motion, London, Washington DC, Tayor \& Francis, 1992.

[7] Malkin, I.G., On the Stability of Motion in the Sense of Lyapunov (in Russian), Mat. Sbornik XXXV, 1938, 47-101.

[8] Markeev, A.P., Dynamics of a Body Being Contiguous to a Rigid Surface (In Russian), Moscow, Nauka, 1992.

[9] Marsden, J.E., Lectures on Mechanics, London Mathematical Society Lecture Note Series 174, Cambridge University Press, 1992.

[10] Zenkov, D.V., A.M. Bloch, \& J.E. Marsden, The Energy-Momentum Method for Stability of Nonholonomic Systems, Dynamics and Stability of Systems, 13, 1998, 123-165.

[11] Zenkov, D.V., A.M. Bloch, \& J.E. Marsden, The Lyapunov-Malkin Theorem and Stabilization of the Unicycle with Rider, preprint, 1999. 